\title{
Conhecimento dos Métodos para Diagnóstico do Câncer de Mama entre Estudantes de Medicina
}

\author{
Knowledge Regarding Breast Cancer Diagnosis \\ Among Medical Students
}

\begin{abstract}
Nilceana Maya Aires, Ruffo de Freitas Júnior,
Regis Resende Paulinelli, Lilian Freire Baêta

Marta Curado C. F. Finotti, Mariluza Terra Silveira
\end{abstract}

\begin{abstract}
RESUMO
Objetivos: avaliar o nivel de conhecimento de noções básicas do diagnóstico do câncer de mama entre os alunos de medicina; promover a discussão entre eles e avaliar o aproveitamento dos alunos do $5^{\circ}$ ano médico, após cursar a Disciplina de Ginecologia. Métodos: por meio de questionários aplicados a 348 alunos do total de 550 alunos, do $1^{\circ}$ ao $5^{\circ}$ ano do curso médico, buscaram-se informações a respeito do conhecimento básico do diagnóstico do câncer de mama. Dos 348 questionários aplicados, 55 (16\%) foram respondidos por estudantes do $5^{\circ}$ ano que haviam cursado a Disciplina de Ginecologia. Destes, 43\% eram mulheres, 62\% tinham familiares médicos e 17\% história familiar de câncer de mama. Resultados: em relação ao conhecimento dos métodos diagnósticos, $84 \%$ dos alunos acertaram o sinal mais freqüente do câncer de mama; 34\%, o melhor método de rastreio; 49\%, quando indicar a mamografia em mulheres assintomáticas; $37 \%$, a periodicidade preconizada da mamografia após 50 anos e 24\%, quando associar o ultra-som à mamografia para detecção do câncer de mama. Os alunos do $5^{\circ}$ ano apresentaram um indice de acerto para todas as perguntas significativamente maior que os demais. O sexo só apresentou diferença em relação ao maior conhecimento pelas mulheres quanto ao melhor periodo para o auto-exame e quando indicar a associação do ultra-som à mamografia. A presença de familiares médicos e a história familiar de câncer de mama não influenciaram as respostas.

Conclusão: mesmo entre acadêmicos de medicina ainda é grande a desinformação a respeito do diagnóstico do câncer de mama. Não obstante, após cursar a disciplina, esse conhecimento aumenta significativamente.
\end{abstract}

PALAVRAS-CHAVE: Mama. Câncer. Mamografia. Ultra-sonografia. Câncer: epidemiologia. Ensino médico.

Departamento de Ginecologia e Obstetrícia da Faculdade de Medicina da Universidade Federal de Goiás.

Correspondência:

Ruffo de Freitas Júnior

Alameda das Rosas, 533, Setor Oeste

74110-060 - Goiânia - GO

Fax: (062) 212-2049

\section{Introdução}

O câncer de mama tem sido uma entidade cada vez mais divulgada e discutida entre os diversos setores da sociedade, não só no Brasil, 
mas em todo o mundo. Parte deste interesse deve-se às altas taxas de incidência e mortalidade da doença ${ }^{2}$ e também às modificações psicossociais acarretadas tanto pelo medo e angústia, quanto pelas modificações provenientes do tratamento ${ }^{4}$.

Apesar da discussão em torno do tema no nosso meio, aproximadamente dois terços dos casos de câncer de mama são diagnosticados nos estádios avançados $^{7}$, situação em que, mesmo se utilizando todos os recursos disponíveis, as taxas de mortalidade são muito altas ${ }^{5}$. Já nos países desenvolvidos, cerca de $70 \%$ dos casos têm sido diagnosticados nos estádios precoces ${ }^{6}$.

Certamente a principal causa dessa situação é a falta de informação. Em recente estudo sobre o auto-exame da mama, conduzido no Hospital das Clínicas da Universidade Federal de Goiás, observou-se que apenas $50 \%$ das pacientes que procuraram o Setor de Mastologia conheciam o auto-exame e somente $30 \%$ das mulheres o praticavam de maneira adequada ${ }^{1}$.

Esses fatores nos levaram a refletir sobre os aspectos da informação junto aos acadêmicos uma vez que estes serão, em sua grande maioria, os responsáveis pela divulgação, independentemente da especialidade a ser seguida no futuro.

Assim, o objetivo deste estudo foi avaliar o nivel de conhecimento sobre noções básicas sobre o diagnóstico do câncer de mama entre os alunos do curso de medicina da Universidade Federal de Goiás, além de promover a discussão entre os acadêmicos de medicina da instituição.

\section{Métodos}

Foram convidados a participar do estudo todos os alunos matriculados no curso médico da Faculdade de Medicina da Universidade Federal de Goiás, por meio do preenchimento de um formulário objetivo, contendo informações a respeito do conhecimento básico do diagnóstico do câncer de mama. Para tal, um questionário foi desenhado especificamente para o presente estudo, contendo informações sobre o aluno e sobre seu conhecimento a respeito do diagnóstico do câncer de mama. Neste formulário não havia nenhum mecanismo que permitisse identificar o aluno que o preencheu.

$\mathrm{Na}$ tentativa de diminuir o número de respostas falsas, em cada questão, manteve-se uma resposta alternativa de desconhecimento sobre o tema (Tabela 1).

O questionário foi aplicado durante a realização dos exames periódicos dos alunos, em diferentes disciplinas. Após consentimento prévio do professor que estava coordenando cada exame, um dos membros do estudo dirigia-se à turma e explicava as finalidades do estudo, sua importância, como preencher o formulário e como entregá-lo posteriormente.

Tabela 1 - Questões aplicadas e as alternativas de resposta.

Qual o melhor período para realizar o auto-exame?

1) Durante a menstruação; 2) 2 a 5 dias após a menstruação*; 3) No período ovulatório; 4) No período pré-menstrual; 5) Em qualquer dia do ciclo;6) Não sei.

Qual o mais freqüente sinal/sintoma do câncer de mama ao exame físico? 1) Mastalgia (dor nas mamas); 2) Nódulo*; 3) Descarga mamilar (saída de secreção pelo mamilo); 4) Linfonodos axilares; 5) Espessamento da pele; 6) Não sei.

Qual o melhor método para rastrear o câncer de mama em mulheres assintomáticas?

1) Auto-exame; 2) Exame físico; 3) Mamografia*; 4) Ultra-som; 5) Ressonância magnética; 6) Não sei.

Quando indicar o uso da mamografia em mulheres assintomáticas

1) Aos 20 anos; 2) Aos 30 anos; 3) Aos 40 anos*; 4) Aos 50 anos; 5) Aos 60 anos; 6) Não sei

Qual a periodicidade da mamografia após os 50 anos?

1) Semestral; 2) Anual*; 3) Bienal; 4) Trienal; 5) Não sei.

Qual a principal indicação do ultra-som associado à mamografia na detecção do câncer de mama?

1) Em mamas volumosas; 2) Em mamas pequenas; 3) Em mamas jovens e/ ou densas*; 4) Em mamas substituídas por gordura; 5) Não sei.

$\mathrm{O} *$ indica a resposta considerada correta para cada pergunta

Após devolvidos, os questionários preenchidos foram arquivados com um dos membros do estudo e, posteriormente, todos os dados foram digitados para o banco de dados.

Após avaliação da consistência dos dados, foram utilizadas freqüências e as respostas das questões, cruzadas com as características dos alunos. Para fins de análise consideraram as taxas de respostas corretas e as taxas de respostas incorretas foram associadas às respostas de desconhecimento. A idade e o sexo do aluno e se ele possuía algum familiar médico ou alguém na família com câncer de mama foram as variáveis independentes, sendo cruzadas com as respostas sobre o conhecimento do câncer de mama.

Utilizou-se o teste do $\chi^{2}$ e $t$ de Student quando cabiveis. Considerou-se o nivel de significância de 95\% $(\mathrm{p}<0,05)$. 


\section{Resultados}

Foram aplicados 550 questionários, dos quais 348 foram preenchidos adequadamente pelos alunos e devolvidos para um dos membros do estudo. A freqüência e a distribuição percentual de respostas corretas, incorretas e não-sabidas, dadas para cada pergunta, são mostradas na Tabela 2 .

Tabela 2 - Distribuição das respostas dos alunos de acordo com as perguntas formuladas.

\begin{tabular}{|c|c|c|c|c|}
\hline \multirow[t]{2}{*}{ Pergunta } & \multicolumn{4}{|c|}{ Resposta } \\
\hline & $\begin{array}{l}\text { Correta } \\
\text { n (\%) }\end{array}$ & $\begin{array}{l}\text { Não Sabe } \\
\text { n (\%) }\end{array}$ & $\begin{array}{l}\text { Incorreta } \\
\text { n (\%) }\end{array}$ & $\begin{array}{l}\text { Total } \\
\text { n (\%) }\end{array}$ \\
\hline Qual o melhor período para auto-exame? & $192(55)$ & $86(25)$ & $70(20)$ & $348(100)$ \\
\hline Qual o sinal mais freqüente do câncer de mama? & $294(85)$ & $28(8)$ & $26(7)$ & $348(100)$ \\
\hline Qual o melhor método de rastreio do câncer de mama? & $118(34)$ & $42(12)$ & $188(54)$ & $348(100)$ \\
\hline $\begin{array}{l}\text { Quando indicar mamografia em mulheres } \\
\text { assintomáticas? }\end{array}$ & $171(49)$ & $75(22)$ & $102(29)$ & $348(100)$ \\
\hline Qual a periodicidade da mamografia após os 50 anos? & $131(38)$ & $83(24)$ & $134(38)$ & $348(100)$ \\
\hline Quando associar o ultra-som à mamografia? & $84(24)$ & $163(47)$ & $101(29)$ & $348(100)$ \\
\hline
\end{tabular}

Foram cruzadas as variáveis independentes com as perguntas ministradas. Em relação ao ano do curso médico freqüentado pelo aluno, observamos que entre os estudantes do $5^{\circ}$ ano, que já haviam passado pela Disciplina de Ginecologia e Obstetrícia, na qual são ministradas as aulas de patologia mamária, houve maior quantidade de acertos referentes às perguntas quanto ao melhor periodo para o auto-exame, melhor método para rastreamento do câncer, quando indicar a mamografia, periodicidade da mamografia e quando associar o ultra-som à mamografia (Tabela 3).

Tabela 3 - Conhecimento do diagnóstico do câncer de mama entre os acadêmicos de medicina de acordo com o ano do curso médico.

\begin{tabular}{|c|c|c|c|c|}
\hline & \multicolumn{2}{|c|}{ Ano do Curso Médico } & \multirow{3}{*}{$\chi^{2}$} & \multirow{3}{*}{$\mathrm{p}$} \\
\hline & $1^{\circ}$ ao 4 anos & $5^{\circ}$ ano & & \\
\hline & $\mathrm{n}(\%)$ & $\mathrm{n}(\%)$ & & \\
\hline Melhor período p/ auto-exame & $148(50)$ & $44(80)$ & 18,1 & $<0,0002$ \\
\hline Sinal mais freqüente & $248(85)$ & $46(84)$ & 0,4 & $<0,9$ \\
\hline Melhor método $\mathrm{p}$ / rastreamento & $95(32)$ & $23(42)$ & 6,9 & $<0,04$ \\
\hline Quando indicar mamografia & $127(43)$ & $44(80)$ & 27,2 & $<0,000002$ \\
\hline Periodicidade da mamografia & $92(31)$ & $39(71)$ & 33,3 & $<0,0000001$ \\
\hline Quando associar o ultra-som & $50(17)$ & $34(62)$ & 52,6 & $<0,00000001$ \\
\hline
\end{tabular}

Em relação ao sexo, observou-se que as estudantes com maior freqüência acertaram as respostas sobre o melhor período para o auto- exame, à periodicidade adequada de realização da mamografia e quando associar o ultra-som à mamografia. As respostas às perguntas para o sinal mais freqüente do câncer, o melhor método para o rastreamento do câncer e quando indicar a mamografia apresentaram porcentagem de acerto igual em ambos os sexos (Tabela 4).

Tabela 4 - Conhecimento do diagnóstico do câncer de mama entre os acadêmicos de medicina de acordo com o sexo.

\begin{tabular}{lccccc}
\hline & Masculino & Feminino & $\chi^{2}$ & $\mathrm{p}$ \\
\cline { 2 - 3 } & $\mathrm{n}(\%)$ & $\mathrm{n}(\%)$ & & \\
\hline Melhor período p/ auto-exame & $81(41)$ & $111(74)$ & 39,5 & $<0,0000001$ \\
Sinal mais freqüente & $169(85)$ & $125(84)$ & 2,1 & $<0,4$ \\
Melhor método p/ rastreamento & $65(33)$ & $53(36)$ & 2,7 & $<0,3$ \\
Quando indicar mamografia & $92(46)$ & $79(53)$ & 2,8 & $<0,3$ \\
Periodicidade da mamografia & $66(33)$ & $65(44)$ & 3,9 & $<0,05$ \\
Quando associar o ultra-som & $38(19)$ & $46(31)$ & 10,1 & $<0,01$ \\
\hline
\end{tabular}

O fato do aluno possuir familiar médico não influenciou nenhuma das respostas (Tabela 5); tampouco a existência de algum familiar com câncer de mama influenciou os índices de acerto para todas as indagações (Tabela 6).

Tabela 5 - Conhecimento do diagnóstico do câncer de mama entre os acadêmicos de acordo com o fato de possuir familiar médico ou não.

\begin{tabular}{lcccc}
\hline & Sim & Não & & \\
\cline { 2 - 4 } & $\mathrm{n}(\%)$ & $\mathrm{n}(\%)$ & $\chi^{2}$ & $\mathrm{p}$ \\
\hline Melhor período para auto-exame & $115(53)$ & $77(58)$ & 0,9 & $<0,4$ \\
Sinal mais freqüente & $186(86)$ & $108(82)$ & 1,2 & $<0,6$ \\
Melhor método para rastreamento & $72(33)$ & $46(35)$ & 0,4 & $<0,9$ \\
Quando indicar mamografia & $100(46)$ & $71(54)$ & 1,8 & $<0,2$ \\
Periodicidade da mamografia & $74(34)$ & $57(43)$ & 2,8 & $<0,1$ \\
Quando associar o ultra-som & $48(22)$ & $36(27)$ & 1,5 & $<0,5$ \\
\hline
\end{tabular}

Tabela 6 - Conhecimento do diagnóstico do câncer de mama entre os acadêmicos de medicina de acordo com o fato de possuir familiar com câncer de mama ou não.

\begin{tabular}{lccccc}
\hline & Sim & Não & & \\
\cline { 2 - 3 } & $\mathrm{n}(\%)$ & $\mathrm{n}(\%)$ & & $\chi^{2}$ & $\mathrm{p}$ \\
\hline Melhor período p/ auto-exame & $34(59)$ & $158(54)$ & 0,4 & $<0,9$ \\
Sinal mais freqüente & $50(86)$ & $44(84)$ & 0,5 & $<0,8$ \\
Melhor método p/ rastreamento & $20(34)$ & $98(34)$ & 0,2 & $<0,9$ \\
Quando indicar mamografia & $29(50)$ & $142(49)$ & 0,3 & $<0,9$ \\
Periodicidade da mamografia & $21(36)$ & $110(38)$ & 0,7 & $<0,8$ \\
Quando associar o ultra-som & $13(22)$ & $71(24)$ & 2,2 & $<0,4$ \\
\hline
\end{tabular}


Questionou-se, por fim, se a divulgação de informações sobre o diagnóstico do câncer de mama estaria sendo suficiente e 128 alunos (37\%) responderam que sim, ao passo que 220 alunos $(63 \%)$ acharam que esta divulgação tem sido feita de maneira inadequada e insuficiente.

\section{Discussão}

Existe um grande desconhecimento nos diversos setores e camadas da sociedade a respeito do câncer de mama e do seu diagnóstico precoce. Acredita-se que em parte isso seja decorrente do descaso e da falta de conhecimento dos profissionais da área de saúde que, por sua vez, não informam ou esclarecem a população a respeito do tema, sendo estas informações divulgadas, quase que exclusivamente, pelos meios de comunicação.

Desta forma, tornam-se necessárias discussões a respeito do conhecimento dos métodos diagnósticos e diagnóstico precoce do câncer de mama, inicialmente dentro da própria faculdade de medicina e, posteriormente, em outros setores, não só no meio universitário, como nas outras camadas da população.

Este estudo mostra que dois terços dos alunos do curso de medicina acham que a divulgação do diagnóstico precoce do câncer de mama é inadequada e insuficiente. Não obstante, a cada ano ocorre, na cidade de Goiânia, local onde foram aplicados os questionários, uma série de encontros científicos, jornadas e congressos, tanto dentro da Universidade quanto fora dela, sempre abordando temas relacionados ao câncer de mama e ao seu diagnóstico. Por razões que fogem ao objetivo deste trabalho, a maioria destes encontros científicos conta com número reduzido de estudantes do curso de medicina. Este fato sugere que, no momento, devam ser criadas novas formas de abordagem do tema para o aluno do curso médico, motivando-o para que possa estar mais interessado nos assuntos referentes ao diagnóstico precoce do câncer de mama, à saúde coletiva e às suas conseqüências dentro da nossa sociedade. É possivel que, com novas abordagens e nova estratégia, haja uma motivação maior, fazendo com que os atuais alunos, futuros profissionais e formadores de opinião, possam estar adequadamente treinados a responder várias dúvidas que surgirem nas diversas comunidades onde os mesmos desenvolverão suas atividades profissionais, favorecendo e facilitando a informação para toda a população.

Outro fato que chamou a atenção foi o baixo percentual de respostas adequadas, principalmente quanto ao melhor método de rastreio do câncer de mama e quanto à periodicidade da mamografia após os 50 anos. Para a primeira, boa parte dos alunos imaginou que o melhor método para rastrear o câncer de mama fosse o auto-exame das mamas, uma vez que, pela facilidade e comodidade, esse método tem sido divulgado com uma certa freqüência na mídia. Em relação à periodicidade da mamografia após os 50 anos, a insegurança na resposta adequada dos alunos basicamente reflete a grande discussão entre os profissionais ligados à patologia mamária sobre se deva ser realizada anual ou bienalmente ${ }^{3}$.

Também chamou a atenção o fato de apenas $55 \%$ dos alunos acertarem a resposta a indagação de quando seria o melhor momento para realização do auto-exame, uma vez que esse método, além de simples, tem sido divulgado por toda a mídia falada, televisionada e escrita. Entretanto, não foi surpresa que as estudantes do sexo feminino conhecessem de maneira mais adequada a questão do momento correto para a realização do mesmo.

Em relação a quando indicar mamografia para mulheres assintomáticas, observou-se que apenas metade do grupo estudado acertou a resposta. Uma vez mais, possivelmente esse baixo índice de acerto se deva à divergência que também existe no meio profissional, no qual várias instituições tentam mostrar, por meio de seus trabalhos, que a mamografia deveria ser iniciada aos 35 anos, aos 40 anos ou mesmo aos 50 anos, conforme resultado dos vários programas de screening ${ }^{3}$.

Quanto à pergunta sobre o sinal mais freqüente do câncer de mama, houve uma alta taxa de acertos para essa resposta (85\%), sendo que esta não foi influenciada nem mesmo pelo ano no curso médico, refletindo possivelmente o conhecimento que a grande massa de nossa população universitária tem sobre a apresentação mais corriqueira da neoplasia mamária.

Em relação às respostas referentes à associação do exame de ultra-som ao exame mamográfico, talvez a complexidade de se envolverem dois exames complementares de imagem levou ao índice mais baixo de respostas corretas (24\%).

Quanto às variáveis independentes, foi observado que para todas as questões, exceto para o sinal mais freqüente do câncer de mama, os alunos que já haviam cursado a Disciplina de Ginecologia e Obstetrícia (na qual são ministradas as aulas sobre patologia mamária) responderam corretamente com maior freqüência que os alunos do $1^{\circ}$ ao $4^{\circ}$ ano, que ainda não cursaram a disciplina. Esse fato mostra a importância da administração das aulas no curso médico. Não obstante, mostra que mesmo após os alunos cursarem a disciplina, boa parte ainda mantém um conhecimento inadequado a respeito de perguntas simples, como, por exemplo, o melhor 
método para o rastreamento do câncer de mama, e também quando associar o ultra-som ao exame mamográfico. Esse fato nos leva a pensar que algumas dúvidas dos alunos persistem e que devem, de alguma maneira, ser melhor abordadas pelos professores ligados à área.

Em relação ao sexo do estudante, avaliandose as respostas para as várias perguntas, não foi surpresa que estudantes do sexo feminino conhecessem mais sobre o auto-exame do que os do sexo masculino. Porém, nos chamou a atenção o fato de as estudantes do sexo feminino conhecerem mais a respeito da periodicidade da mamografia e também quanto à associação do ultra-som ao exame mamográfico. Talvez essas duas diferenças possam ser justificadas pelo maior interesse pessoal das mulheres em relação ao tema.

Ao desenhar esse estudo, acreditávamos que o fato de o acadêmico de medicina possuir algum familiar médico, ou então alguém na família com o diagnóstico pregresso de câncer de mama, pudesse favorecer, de alguma maneira, aquele aluno nas suas respostas. Apesar da alta freqüência de acadêmicos com familiares médicos e também daqueles que apresentaram familiares com câncer de mama, para nossa surpresa, não houve diferença significativa em nenhuma das respostas às várias perguntas.

Assim, concluímos que o conhecimento do diagnóstico do câncer de mama entre os estudantes de medicina da Universidade Federal de Goiás é precário, com uma alta taxa de desconhecimento entre os acadêmicos, principalmente no tocante ao auto-exame da mama, à mamografia e ao exame de ultra-sonografia. Os próprios acadêmicos acreditam que a informação passada a eles seja inadequada e que esta orientação deva ser feita de maneira mais efetiva, para que se sintam mais motivados na busca do conhecimento sobre esse tema. Não obstante, fica a dúvida de como propiciar informações de maneira mais adequada, não só para todos os acadêmicos de medicina, mas também para outros universitários e para a comunidade de uma maneira geral. Fica por serem definidas quais seriam as formas mais adequadas para que esse conhecimento possa ser transmitido aos vários segmentos dentro e fora da Universidade.

\section{SUMMARY}

Purpose: to evaluate how knowledgeable medical students at the Universidade Federal de Goiás were concerning the basic diagnostic principles breast cancer. The study also aimed at promoting a debate among the students and at assessing the understanding of the students in the fifth year of medical school, who had already attended the Gynecology course. Methods: Through questionnaires given to 348 individuals, from the first to the fifth year, out of a total population of 550 students, the authors searched for information with regard to basic knowledge on the diagnosis of breast cancer. Of the 348 questionnaires, 55 (16\%) were given to fifth-year students, who had already attended the Gynecology course. Furthermore, $43 \%$ of the students were women, $62 \%$ had medical doctors in their immediate family, and $17 \%$ had a family history of breast cancer.

Results: in regard to the knowledge of diagnostic methods, $84 \%$ of the students were aware of the most frequent sign of breast cancer, 34\% knew which was the best screening method, $49 \%$ knew when to refer asymptomatic women to mammography, 37\% knew the recommended interval between mammography for women above the age of 50 , and $24 \% \mathrm{knew}$ when to associate ultrasound to mammography for the detection of breast cancer. The fifth-year students provided correct answers at a significantly higher rate, when compared to the others. Concerning gender, the only difference regarded the fact that women showed a better knowledge as to the best time for self-examination and when to recommend ultrasound associated with mammography. The presence of medical doctors in the family and a history of family members with breast cancer did not have any influence on the answers.

Conclusion: The lack of information in regard to the diagnosis of breast cancer is very high, even among medical students. Nevertheless, the rate of information increases significantly after students are taught Gynecology, which is only offered during the fifth year of the medical school.

KEY WORDS: Breast cancer: diagnosis. Mammography. Ultrasonography. Medical teaching.

\section{Referências}

1. Freitas Jr R, Soares VF, Melo NF, Andrade ML, Philocreon GR. Fatores determinantes do conhecimento e prática do auto-exame de mama. Rev Bras Ginecol Obstet 1996; 18: 387-91.

2. Hardy EE, Pinotti JA, Faundes A. Variações internacionais da incidência e da mortalidade por câncer de mama. Femina 1983; 11: 106-15.

3. Kerlikowske K, Grady D, Rubin SM, Sandrock C, Ernster VL. Efficacy of screening mammography. A meta-analysis. JAMA 1995; 273: 149-54.

4. Magarey CJ. Aspects of the psychological management of breast cancer. Med J Australia 1988; 148: 239-42.

5. Piccart MJ, Valeriola D, Paridaens R, Balikdjian D, Mattheiem WH, Loriaux C et al. Six-year of a multimodality treatment strategy for locally advanced breast cancer. Cancer 1988; 62: 2501-6.

6. Ries L. Breast cancer stage distribution. J Natl Cancer Inst 1995; 87: 1435.

7. Silveira Jr LP, Freitas Jr R, Carneiro AB, Ribeiro LFJ, Queiroz GS. Fatores socio-demográficos associados com o estadiamento das pacientes com câncer de mama. Rev Bras Ginecol Obstet 1996; 18: 411-5. 